\title{
Variación del contenido de nitrito de sodio residual en diferentes lotes de salchichas, de una misma formulación de una empresa productora costarricense
}

\author{
Variation of residual sodium nitrite content in different sausage batches based \\ on the same formula in a Costa Rican company
}

\author{
Laura Vindas Angulo ${ }^{1} \quad$ Nazareth Rodríguez Arce 2 \\ Yorleny Araya Quesada ${ }^{3}$
}

Recibido: 11/8/2016 / Aprobado: 7/3/2017

Resumen

\begin{abstract}
El nitrito de sodio o potasio es uno de los ingredientes principales para la elaboración de productos embutidos o carne procesada ya que desempeña un papel fundamental en su preservación e influye en las características sensoriales. Como parte de la regulación y el control del nitrito en dichos productos, en Costa Rica, existe el Reglamento Técnico RTCR 411:2008 Productos Cárnicos Embutidos: Salchicha, Salchichón, Mortadela y Chorizo, estipula que deben poseer una cantidad menor a $125 \mathrm{mg} / \mathrm{kg}$ de nitrito de sodio o potasio. En la presente investigación se realizó un estudio del contenido de nitritos, en 30 lotes de salchichas, de una misma empresa productora en Costa Rica con una misma formulación. Se obtuvo que el promedio de nitrito de sodio fue de $88 \pm 6 \mathrm{mg} / \mathrm{kg}$, siendo $121 \pm 2 \mathrm{mg} / \mathrm{kg}$ el resultado más alto y $38 \pm 2 \mathrm{mg} / \mathrm{kg}$ el del menor valor para los lotes. Tales resultados evidencian que ninguna de las muestras infringe la reglamentación costarricense; no obstante, su comportamiento es fluctuante en las concentraciones de nitritos de los distintos lotes, se plantea la necesidad de estudiar cuáles son factores que influyen para controlarlos con el fin de reducir la fluctuación.
\end{abstract}

Palabras clave: salchichas, embutidos, carne procesada, nitritos de sodio o potasio, curado de productos embutidos, carcinógeno.

\section{Abstract}

Sodium nitrite or potassium is one of the main components to produce sausage or processed meat. It is an important ingredient to preserve and to stimulate taste buds. World Health Organization (October 2015) has reported that processed meat is classified as a carcinogen; there is evidence that makes a correlation with colorectal cancer in human beings consuming them. As part of the regulation and control of nitrite in Costa Rica, there are technical restrictions (Reglamento Técnico RTCR 411:2008 Productos Cárnicos Embutidos: Salchicha, Salchichón, Mortadela y Chorizo), it stipulates that it must have fewer than $125 \mathrm{mg} / \mathrm{kg}$ of sodium or potassium nitrite in the products. This study shows a content of nitrite in 30 lots of sausages with the same formula, in the same production. It was found that the average of sodium nitrite was $88 \pm 6 \mathrm{mg} / \mathrm{kg}$, being $121 \pm 2 \mathrm{mg} / \mathrm{kg}$ the highest result and $38 \pm 2 \mathrm{mg} / \mathrm{kg}$ for the lower value of the lots. The results show that none of the samples has broken any regulations. On the other hand, the concentrations of nitrite perform fluctuating in different lots due to variables or process steps that should be improved to reduce fluctuation because it not depends on the standard formula. It depends on different factors like chemical product variables, stages of the process, and curing salt. They must be under a strict control, nitrites especially.

Key words: sausages, processed meat, sodium or potassium nitrite, cured sausage products, carcinogen.

\footnotetext{
${ }^{1}$ Magister en Sistemas Modernos de Manufactura, bachiller en Química. Profesora e investigadora del Departamento de Ciencias Naturales, Sede de Occidente, Universidad de Costa Rica. Correo electrónico: laura.vindasangulo@ucr.ac.cr

${ }^{2}$ Bachiller en Laboratorista Químico. Sede de Occidente, Universidad de Costa Rica. Correo electrónico: nazareth9304gmail.com ${ }^{3}$ Magister en Gerencia Agroempresarial, licenciada en Tecnologías de Alimentos. Docente en la Escuela de Tecnología de Alimentos, Universidad de Costa Rica. Correo electrónico: yorleny.araya@ucr.ac.cr
} 


\section{Introducción}

La Organización Mundial de la Salud (OMS) (2015), define la carne procesada o embutidos como la carne que se ha transformado a través de la salazón, el curado, la fermentación, el ahumado $\mathrm{u}$ otros procesos para mejorar el sabor o la conservación. Los embutidos son preparados a partir de carne picada, grasa, sal, condimentos, fosfatos (como reguladores de acidez), eritorbato y otros aditivos conservantes que se introducen en fundas, algunos ejemplos de estos tipos de productos son las salchichas, salchichón, chorizo, y mortadela.

Los embutidos son sometidos a un proceso de curado, en el cual se adiciona nitrito de sodio (NaNO2) o potasio (KNO2), en forma pura o mezclado con sal común (cloruro de sodio) denominado sal de cura. La aplicación original de la sal de cura estaba relacionada con el desarrollo del color rosado estable, además, genera características sensoriales de textura, aroma y sabor. Los nitritos retardan la oxidación lipídica, una de las causas de pérdida de calidad de las carnes (Bazan, 2008; Sánchez, 2008, Alba, 2008; Jiménez y Blázquez, 2009; Andújar, 2009 y Martin, 2012).

Respecto de la alteración oxidativa, Morales y Repetto (2012), mencionan que se produce como consecuencia de la acción del oxígeno atmosférico sobre las grasas (fundamentalmente las insaturadas) de forma espontánea (autoxidación); es la principal vía de modificación de aceites, grasas y alimentos lipídicos, ya que se produce una serie de reacciones en cadena, que tienen lugar a través de radicales libres, lo cual genera una gama de nuevos compuestos que se diferencian tanto en sus pesos moleculares como en sus polaridades. Además, los mismo autores agregan que la oxidación es la causa más importante de la pérdida de calidad del alimento desde el punto de vista nutricional, esto se debe, fundamentalmente, a la formación de nuevos compuestos (principalmente compuestos oxidados y poliméricos) por modificación de los ácidos grasos insaturados constituyentes de los lípidos, a la interacción de los lípidos oxidados con las proteínas, a la oxidación paralela de vitaminas con actividad antioxidante, entre otros; que disminuyen sensiblemente su valor nutritivo, incluso en alimentos de bajo contenido graso.

Con el curado se asegura la preservación, pues la sal y el nitrito, junto con el tratamiento térmico comúnmente aplicado a los productos curados, inhiben el crecimiento de bacterias, que causan alteraciones en la carne, y la germinación de esporas bacterianas; inhibe la producción de la toxina de la bacteria Clostridium botulinun, la cual causa una rara, pero seria enfermedad llamada Botulismo (Pérez, Andújar y Venegas, 2009). La efectividad del nitrito para controlar la producción de la toxina botulínica está demostrada y se ha encontrado que diversos factores como $\mathrm{pH}$, contenido de sal y tratamiento térmico influyen en su eficacia (Cui, Gabriel y Nakano, 2010). Además del curado, los embutidos se pueden someter al proceso de cocción previo a la venta y se almacenan en refrigeración. Por tratarse de productos cocidos son alimentos muy usados. Jimenez-Robles (2014) señala que los consumidores consideran que los embutidos son prácticos y convenientes, razones que motivan su consumo en diferentes platillos y ocasiones.

A pesar de los beneficios en el curado de la carne, el nitrito es tóxico en dosis elevadas, Martín (2001), señala que la dosis letal de nitrito es de 300 $\mathrm{mg} / \mathrm{kg}$ de peso corporal, por esta razón, se evita en, forma general, su uso en forma pura, y se añade a los productos cárnicos diluido en sal lo que se denomina sal de cura. En Europa, es común que la sal usada en la industria cárnica contenga 0,5 - 0,6 $\%$ de nitrito de sodio, de esta sal se añade alrededor de 1,5 - 2,0 \% de sal, esto representa una adición de entre 75 y 100 ppm o mg/kg de nitrito (Pérez, Andújar y Venegas, 2009).

Por otra parte, Ordóñez (2007), recalca que el uso de nitratos y nitritos en la elaboración de productos cárnicos curados conlleva la producción de $\mathrm{N}$-nitrosaminas que se forman por nitrosación ${ }^{2}$ de aminas y amidas y otros compuestos 
nitrogenados. Los $\mathrm{N}$-nitrosocompuestos son agentes teratógenos, mutágenos y probables carcinógenos, altamente peligrosos para la salud humana (Antón y Lizaso, 2001). Las $\mathrm{N}$-nitrosaminas pueden surgir mediante síntesis endógena (en el organismo, fundamentalmente en la saliva y el estómago) y por formación exógena (en el producto dependiendo de diversos factores que, en el caso de los alimentos, el más importante es el tratamiento térmico aplicado en el cocinado, fritura, asado, cocción, etc.). Por ello, la utilización de estas sales está regulada y tiene establecida una dosis máxima en función del tipo de producto elaborado (Serrano-Pérez, de OnaBaquero, \& Orts-Laza, 2012).

Respecto de la síntesis endógena, Antón y Lizaso (2001), señalan que bajo circunstancias específicas, como la gastritis crónica, los nitritos pueden oxidarse en el estómago a agentes nitrosantes $(\mathrm{N} 2 \mathrm{O} 3, \mathrm{~N} 2 \mathrm{O} 4)$ y reaccionar para formar
$\mathrm{N}$-nitrosocompuestos; lo que a su vez puede dar inicio al desarrollo de células cancerígenas en el organismo de los consumidores de productos embutidos.

Por esta razón, la Organización Mundial de la Salud (2015), comunicó que la carne procesada ha sido clasificada como carcinógena para los humanos (Grupo $1^{3}$ ), basada en evidencia suficiente en humanos de que su consumo causa cáncer colorrectal.

Estudios llevados a cabo por la Agencia Internacional de Investigación sobre el Cáncer (IARC por su sigla en inglés), han determinado que el nitrito posee efectos negativos para la salud de los consumidores; al punto de que la OMS (2015), informara que la carne procesada es clasificada como carcinógena, esta afirmación se basa en
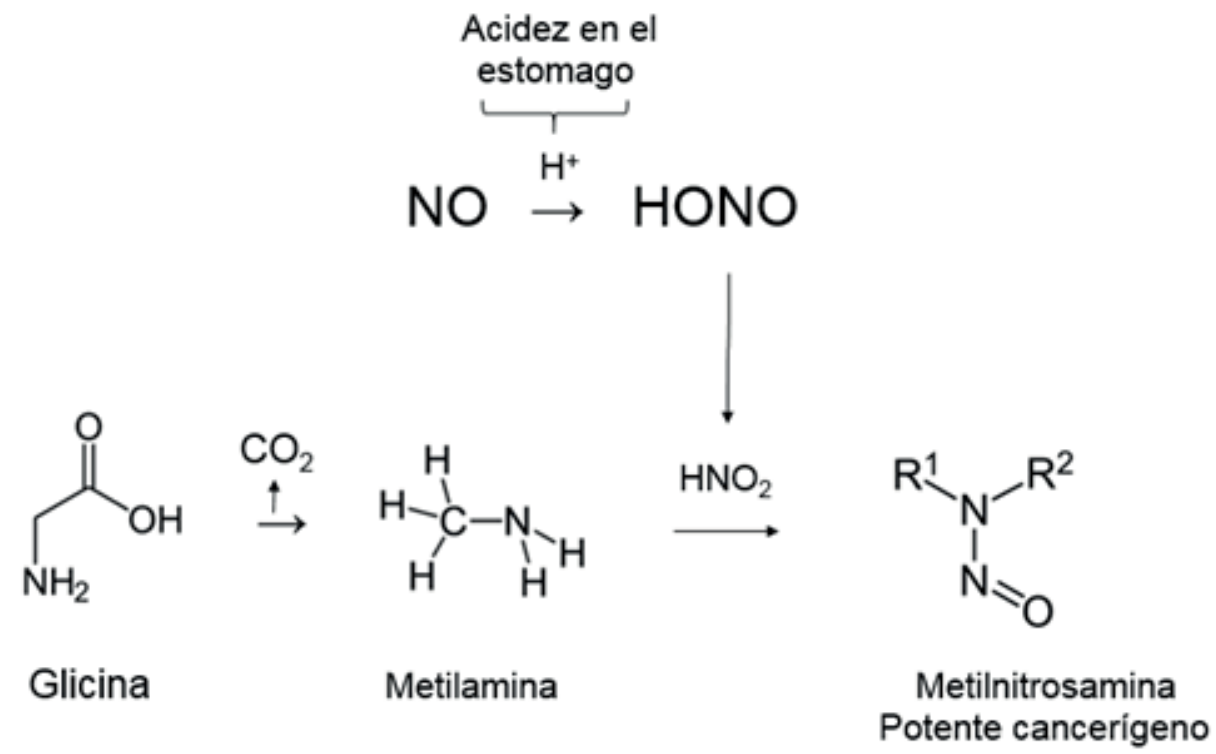

Figura 1. Formación de metilnitrosamina en el estómago en presencia de nitritos. R1 representa un grupo metil (CH3) y R2 un hidrógeno (H).

Fuente: elaboración propia, marzo 2016.

\footnotetext{
${ }^{2}$ Proceso que convierte un compuesto orgánico en derivados nitrosos.

${ }^{3}$ Grupo 1: "carcinógeno para el ser humano" Hay pruebas suficientes que confirman que puede causar cáncer a los humanos. Definición de la Agencia Internacional de Investigación sobre el Cáncer (IARC por su sigla en inglés).
} 
evidencia suficiente en humanos que demuestran que el consumo de carne procesada provoca cáncer colorrectal; e incluso, el estudio concluyó que cada porción de 50 gramos de carne procesada consumida diariamente aumenta el riesgo de cáncer colorrectal en un $18 \%$; dicho riesgo se acrecienta conforme se incrementa el consumo de estos productos.

Igualmente, dicho comunicado de la OMS (2015), indica que "en vista del gran número de personas que consumen carne procesada, el impacto global sobre la incidencia del cáncer es de importancia para la salud pública"; por lo que es recomendable que se busquen nuevas alternativas de aditivos que reemplacen los nitritos de sodio o potasio y que los consumidores conozcan con claridad los efectos que puede tener el consumo de productos embutidos en su salud.

En el presente estudio, se realizó un análisis del contenido de nitritos en diferentes lotes de salchichas de una misma empresa productora, con la finalidad de evaluar las concentraciones y el comportamiento en diversos lotes de una misma formulación. De igual manera, se determinó si cumplen con lo establecido en el Reglamento técnico RTCR 411:2008 Productos Cárnicos Embutidos, el cual indica que los productos embutidos no deben sobrepasar los $125 \mathrm{mg} / \mathrm{kg}$ de nitrito de sodio o potasio.

Es importante resaltar que el Reglamento técnico RTCR 411:2008 Productos Cárnicos Embutidos, No 35079-MEIC-MAG-S, tiene como objetivo establecer las especificaciones físicas, químicas y sanitarias que deben cumplir los productos cárnicos denominados como salchicha, salchichón, mortadela y chorizo, que se comercializan en el país.

\section{Materiales y métodos}

El estudio, se realizó en el Laboratorio de Química del Recinto de Grecia, de la Universidad de Costa Rica, durante los meses de octubre de 2015 a febrero de 2016. Las salchichas que se analizaron son las más consumidas a nivel nacional, según la Cámara Costarricense de Embutidores y Procesadores de Carne, dato proporcionado en el mes de mayo del $2015^{4}$. Se escogió una única marca de salchichas con el fin de asegurar estandarización de proceso e ingredientes y determinar la variación en el nitrito residual, aspecto importante que servirá como base para estudios posteriores.

Los muestreos se realizaron en diferentes supermercados ubicados en el cantón de San Ramón, Alajuela; se analizaron 30 lotes distintos del mismo embutido; además, se trituraban entre cuatro o cinco salchichas por lote, escogidas al azar, y de allí se tomaba la masa respectiva para el análisis químico. Los muestreos de los lotes de salchichas se llevaron a cabo por medio del procedimiento de muestreo a juicio, metodología empleada por los entes nacionales encargados de la regulación de estos productos: el Servicio Nacional de Salud Animal (SENASA) y el Ministerio de Salud (quien da la orden de realizar los análisis de estos alimentos para su control en el mercado nacional). Además, porque sin importar el paquete de salchichas que se escoja, sin excepción, deben cumplir con los valores estipulados en el Reglamento Técnico RTCR: 4112008.

El método para el análisis de nitritos en embutidos empleado es el indicado en el Reglamento Técnico RTCR 411:2008 Productos Cárnicos Embutidos: Salchicha, Salchichón, Mortadela y Chorizo, el cual corresponde a un ensayo oficializado por la Official Methods of Analysis AOAC 973.31 (AOAC, 2005).

\footnotetext{
${ }^{4}$ Reunión con el Servicio Nacional de Salud Animal (SENASA) y la Cámara Costarricense de Embutidores y Procesadores de Carne, 15 de mayo de 2015.
} 


\section{Resultados}

A partir de las determinaciones analíticas realizadas se obtuvieron los siguientes resultados promedio para cada lote de muestras de salchichas (Cuadro 1).

En la figura 2 se muestra el comportamiento del contenido de nitritos con un intervalo de confianza de $\alpha=0,05$; para los diferentes lotes analizados, donde se puede evidenciar datos muy cercanos al valor establecido como límite máximo permisible, resaltando el valor de $121 \pm 2 \mathrm{mg} / \mathrm{kg}$; y no dejando de lado, que hay siete lotes con valores superiores a los $100 \mathrm{mg} / \mathrm{kg}$ de nitrito de sodio presentes en las muestras, los cuales evidencian datos próximos al permitido. De igual forma, hay otros resultados menores, incluso inferiores a los 50 $\mathrm{mg} / \mathrm{kg}$; como es el caso de la muestra con lote 19 que presentó un valor de $38 \pm 2 \mathrm{mg} / \mathrm{kg}$.

El contenido de nitrito mínimo detectado para estas muestras fue de $38 \mathrm{mg} / \mathrm{kg}$, el máximo de $121 \mathrm{mg} / \mathrm{kg}$, con un promedio de $88 \pm 21,3 \mathrm{mg} /$ $\mathrm{kg}$ (Cuadro 2).

Cuadro 1. Resultados del análisis de nitritos de sodio realizado a las muestras de salchichas de una
empresa productora en Costa Rica

Fuente: Elaboración propia, marzo 2016.

\footnotetext{
${ }^{5}$ No se logró cuantificar los nitritos para este lote de salchichas porque el valor de absorbancia obtenido era mayor que los valores de absorbancias de la curva de calibración.
} 


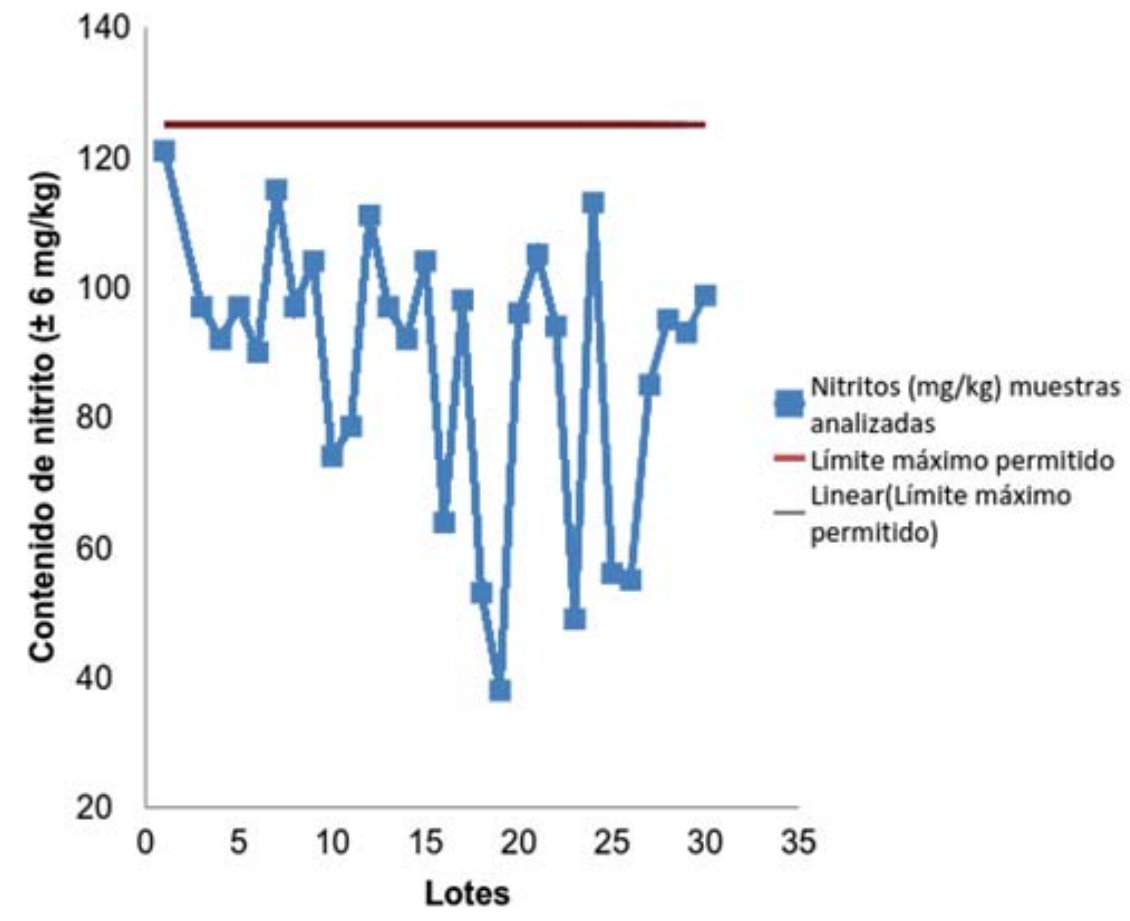

Figura 2. Contenido de nitrito de las muestras analizadas, con su intervalo de confianza para un $\alpha=0,05$, comparado con el límite máximo permitido en el reglamento técnico RTCR 411:2008.

Fuente: Elaboración propia, marzo 2016.

Cuadro 2. Distribución del contenido de nitrito de sodio en muestras de salchichas de una misma formulación comercializadas por una empresa productora costarricense

\begin{tabular}{|cc}
\hline Cuantil & Nitrito de sodio ppm \\
\hline $100 \%$ (Máximo) & 121 \\
$90 \%$ & 113 \\
\hline $75 \%($ Cuartil) & 101,35 \\
\hline $50 \%$ (Mediana) & 95 \\
\hline $25 \%($ Cuartil) & 76,3 \\
\hline $10 \%$ & 53 \\
2,5 & 38 \\
\hline $0 \%($ Mínimo) & 38 \\
\hline
\end{tabular}

Fuente: Elaboración propia, marzo 2016. 


\section{IV.Discusión}

La adición de sustancias a los alimentos es una práctica que viene realizándose desde las civilizaciones más antiguas. El empleo de la sal o la salmuera, así como otros productos (vinagre, vino), se ha utilizado desde la antigüedad para alargar la vida de los alimentos (Serrano-Pérez, de Ona-Baquero, \& Orts-Laza, 2012).

En la industria alimenticia, una gran variedad de productos cárnicos, tales como las salchichas, chorizos, mortadela, salchichón, jamón serrano, entre otros; se someten a procesos como el curado. El curado consiste en la conservación de la carne, por inhibición del desarrollo microbiano, mediante la adición de sal común, nitrato y/o nitrito sódico y otras sustancias, como, por ejemplo, azúcares, fosfatos, ascorbatos y otras, que contribuyen conjuntamente en la mejora de las características sensoriales del producto embutido (Andújar, 2009; Pérez, Andújar y Venegas, 2009). Debido a que el nitrito de sodio tiene un efecto perjudicial en la salud de los consumidores, genera que su regulación sea un aspecto crítico de salud pública. Para el caso de Costa Rica, el Servicio Nacional de Salud Animal (SENASA) es la institución que se encarga de controlar que los productos embutidos cumplan con el límite establecido en el Reglamento Técnico RTCR 411:2008 Productos Cárnicos Embutidos: Salchicha, Salchichón, Mortadela y Chorizo, el cual indica que deben poseer una cantidad menor a $125 \mathrm{mg} / \mathrm{kg}$ de nitrito de sodio o potasio.

Vindas (2014) señala que las ventas de embutidos en Costa Rica en el canal al detalle pasaron de 20,2 toneladas en el 2008 a 25 toneladas en el 2013, indica también, que esas ventas significaron $\$ 197$ millones, lo que representa un $72 \%$ más que en el 2008 , lo que demuestra que los embutidos son un producto de consumo importante. Además, Jiménez-Robles (2014), agrega que las personas escogen consumir embutidos debido a su versatilidad, pues, se pueden preparar de varias formas, acompañar con una amplia gama de alimentos y porque se puede hacer una comida con estos en poco tiempo. Conjuntamente, adiciona que la disponibilidad de embutidos en diferentes puntos de venta y su variedad logra que se promueva su consumo.
Como se presentó anteriormente, los embutidos son productos ampliamente comercializados en el país, por lo que es importante reiterar que es de suma importancia que los lotes de productos embutidos que salen al mercado cumplan con los parámetros estipulados por el Reglamento Técnico RTCR 411:2008, y que se lleven a cabo análisis para cuantificar el contenido de nitritos y garantizar que sean aptos para el consumo.

En cuanto a los resultados obtenidos para los diferentes lotes de salchichas de una misma empresa productora y de una misma formulación, se aprecia que ninguno infringe el valor máximo de $125 \mathrm{mg} / \mathrm{kg}$ de nitrito establecido en el Reglamento, esto demuestra que la empresa productora toma medidas de control en sus productos y no expone a un mayor riesgo a los consumidores de este embutido en específico.

$\mathrm{Al}$ considerar que las muestras corresponden a un mismo producto de una misma marca, se puede observar (Figura 2) que existen variaciones o fluctuaciones importantes entre lotes, por lo que resulta de especial relevancia efectuar estudios que analicen los factores que tienen influencia en el contenido final de nitritos; esto porque, en el proceso se agrega una cantidad conocida de sal de cura, que genera la reacción de los nitritos con la carne, para así formar el compuesto que da el color característico a los embutidos (nitrosilmioglobina $\mathrm{MbNO}$ o MbFeII NO); pero, al final de su producción queda un contenido de nitrito residual en el producto, lo que hace necesario determinar de qué depende ese nivel.

Merino, Darnerud, Toldrá e Ilbäck (2016), encontraron una importante disminución en el contenido de nitrito entre el momento de adición y una vez transcurridas 24 horas. En periodos posteriores, se siguió observando una disminución, aunque no tan drástica y que varió entre los productos que estudiaron. Pérez, Bosch y García (1996) observaron una disminución del nitrito durante el almacenamiento de salchichas, Karl-Otto Honikel (2008), en su revisión también menciona que el nitrito en los productos almacenados disminuye con el tiempo. Con respecto 
a los resultados de la presente investigación, pudieron ser influenciados por las diferencias que existieron entre la fecha de análisis en el laboratorio de la muestra y el tiempo de producción en la planta de proceso, aspecto que no fue controlado, porque se tenía el objetivo de hacer un diagnóstico de los niveles de nitrito a los que los consumidores se exponen cuando adquieren las salchichas en los puntos de venta. Asimismo, es importante resaltar que otro factor causante de las fluctuaciones, tanto de las muestras dentro de un mismo lote como de las muestra entre diferentes lotes, es la falta de homogeneidad de la sal de cura; ya que mezclar dos líquidos o un sólido en un líquido es muy fácil, pero mezclar dos sólidos es muy difícil; lo que quiere decir que el contenido de nitritos en el embutido no es homogéneo.

Por otra parte, relacionado con los resultados de menor contenido de nitritos, Bazán (2008) menciona que los nitritos a altas concentraciones tienen la capacidad de inhibir el crecimiento de algunos microorganismos aerobios como Pediococcus acidilactici, Lactobacillus plantarum y Staphylococcus carnosus los cuales, a concentraciones mayores de $50 \mathrm{ppm}$ se detiene su crecimiento y comienzan a morir después de un periodo de ocho días de maduración. De los resultados se puede observar que dos lotes se encuentran por debajo de los $50 \mathrm{mg} / \mathrm{kg}$ de NaNO2 (38 2 mg/kg y $49 \pm 1$ $\mathrm{mg} / \mathrm{kg}$ ) y tres lotes están muy próximos a este valor, es importante destacar que las salchichas tiene otras barreras de protección contra microorganismos, por ejemplo, adición de otros antimicrobianos, el empaque y la temperatura de almacenamiento. Las muestras con los niveles más bajos de nitrito estaban próximas a la fecha de vencimiento, por lo que este producto de no ser consumido, se retira de los puntos de venta, esto minimiza las posibilidades de tener producto con altas cargas microbiológicas en el punto de venta.

Por otra parte, es importante resaltar que un $50 \%$ de las muestras se encuentra entre 75 y $100 \mathrm{mg} / \mathrm{kg}$, rango que puede considerarse idóneo para los productos embutidos, considerando que no va a estar tan próximo al valor máximo establecido en el reglamento (Cuadro 2). De lo anterior, es importante resaltar que a pesar de que todos los lotes analizados de esta empresa embutidora, ubicada en Costa Rica, cumplen con la cantidad máxima de nitritos de sodio o potasio que establece el Reglamento Técnico RTCR 411:2008, correspondiente a productos cárnicos embutidos, los consumidores aún así presentan riesgo de desarrollar cáncer colorrectal, y aumentan sus probabilidades si el consumo es excesivo.

Es claro que aún existen diversas variables que pueden tener un impacto en el contenido de nitrito de sodio de los productos embutidos, los cuales pueden estar ligados con las características químicas del producto, las materias primas o ciertas etapas del proceso de elaboración. De igual manera, será un desafío para la industria alimentaria alcanzar productos cárnicos procesados con un nivel mínimo de aditivos o la eliminación de los mismos.

\section{Conclusiones}

- Ningún lote de salchichas analizado en la presente investigación, infringe el valor máximo de $125 \mathrm{mg} / \mathrm{kg}$ de nitrito de sodio establecido en el Reglamento Técnico RTCR 411:2008 Productos Cárnicos Embutidos: Salchicha, Salchichón, Mortadela y Chorizo.

- A pesar de que las salchichas provienen de una fórmula estandarizada, se evidencia que existen fluctuaciones en el contenido de nitrito de sodio del producto final.

- A raíz del amplio consumo de productos embutidos a nivel nacional, es de suma importancia estandarizar el contenido de nitrito de sodio o potasio residual en la formulación, para garantizar al consumidor final un producto inocuo. 


\section{Recomendaciones}

A partir del estudio químico realizado a los lotes del producto embutido, se recomienda lo siguiente:

- Para investigaciones futuras, se recomienda realizar un análisis para identificar cuál o cuáles variables afectan el contenido de nitritos en las salchichas. Dichas variables pueden ser el $\mathrm{pH}$, grasa total, proteína, cenizas, mioglobina, humedad, pigmentos, entre otras.

- Se recomienda a las empresas embutidoras revisar sus sistemas de producción con el objetivo de identificar en qué etapa del proceso sucede el cambio que genera las variaciones en la cantidad de nitrito de sodio.

- Es recomendable analizar las materias primas con las que se formulan los productos embutidos, en este caso, los aditivos que ayudan a su conservación, como es la sal de cura, y no basarse soloamente en lo que indica el certificado de análisis suministrado por el proveedor para determinar si la cantidad de nitrito de sodio es estable en todos los lotes entregados por los diferentes proveedores.

- Las fluctuaciones en el contenido de nitritos para los diferentes lotes de salchichas de una misma empresa productora de embutidos con una formulación estandarizada, demuestran que este parámetro no es constante en la fabricación de estos productos, por lo que existe alguna (as) variable, que aún se desconoce, que genera este impacto.

\section{Referencias}

Alba,N. (2008). Ciencia Tecnología e Industria de Alimentos. Grupo Latino Editores. Colombia.

Andújar, G. (2009). El curado de la carne y la elaboración tradicional de piezas curadas ahumadas. Instituto de Investigaciones para la Industria Alimentaria. Cuba: Editorial Universitaria. pp: 85-87. Recuperado de: http://site.ebrary.com.ezproxy. sibdi. ucr.ac.cr:2048/lib/sibdilibrosp/reader. action?docID $=10317323$

Antón, A. y Lizaso, J. (2001). Nitritos, nitratos y nitrosaminas. Fundación Ibérica para la Seguridad Alimentaria. Recuperado de: http://www.proyectopandora.es/wp-content/ uploads/Bibliografia/13181019_nitritos_ nitratos.pdf

AOAC. (2005). Nitrites in cured meat. Colorimetric Method. AOAC Official Method of Analysis 973.31

Bazán, E. (2008). Nitritos y Nitratos: Su uso, control y alternativas en embutidos cárnicos. Nacameh 2 (2): 160-187. Recuperado de: https://dialnet. unirioja.es/servlet/articulo? codigo $=3664829$

Comisión del Codex Alimentarius. (2003). Programa conjunto $\mathrm{FAO} / \mathrm{OMS}$ sobre normas alimentarias comisión del Codex Alimentarius. Informe de la $35^{a}$ reunión del Comité del Codex sobre aditivos alimentarios y contaminantes de los alimentos. pp: 2.

Ibáñez, F., Torre,P. e Irigoye, A. (2003). Aditivos alimentarios. Recuperado de: http://www. nutricion.org/publicaciones/revista_ agosto_03/Funcionales/aditivos.pdf

Honikel, K.O. (2008). "The use and control of nitrate and nitrite for the processing of meat products". Meat Science. 78:68-76 
Jiménez, A., Araya, Y., Ivankovich, C. y García, M. (2014). "Hábitos de consumo de embutidos en el cantón de San Carlos y el área metropolitana de Costa Rica”. Tecnología en Marcha, 27(4): 113-124. Recuperado de: https://dialnet. unirioja.es/descarga/articulo/5001539.pdf

Jiménez, F. y Blázquez, J. (2009). Additives: Preservatives. Handbook of Processed Meats and Poultry Analysis. CRC Press. Estados Unidos. pp: 100. Recuperado de: http:// www2.fea.unicamp.br/ labcarne/wordpress/ wp-content/uploads/2012/09/2-NOLLET-L.M.-L..pdf

Majul, E., Morón, J. y Ramón, A. (2004). Estimación de la ingesta diaria potencial de nitritos en productos cárnicos de mayor consumo en adolescentes. Respyn 5 (3). Recuperado de: http://www.respyn.uanl.mx/v/3/articulos/ nitiritos.htm

Martin, M. (2001). Meat Curing Technology. En Young, O.A., Roger, R.W., Hui, Y., y Nip, W.K. Meat Science and Applications. CRC Press. Recuperado de: http://www.crcnetbase.com/ doi/abs/10.1201/9780203908082.ch20

Martin, J.M. (2012). Meat-Curing Technology. En Hui, Y.H. Handbook of meat and meat processing. 2 ed. Recuperado de www. crcnetbase.com/isbn/978-1-4398-3683-5

Merino, L., Darnerud, P.O., Toldrá, F. e Ilbäck, N.G. (2016). "Time-dependent depletion of nitrite in pork/beef and chicken meat products and its effect on nitrite intake estimation". Food Additives \& Contaminants. 3(2): 186-192, DOI: $10.1080 / 19440049.2015 .1125530$

Morales, M. y Repetto, M. (2012). Grasas y aceites alimentarios. Toxicología alimentaria. España: Ediciones Díaz de Santos. pp 517537. Recuperado de: http://site.ebrary.com. ezproxy.sibdi.ucr.ac.cr:2048/lib/sibdilibrosp/ reader.action? docID $=10592289$
Organización Mundial de la Salud. (2015). El Centro Internacional de Investigaciones sobre el Cáncer evalúa el consumo de la carne roja y de la carne procesada . Recuperado de: http://www.who. $\mathrm{int} /$ mediacentre/news/releases/2015/cancerred-meat/es/

Ordóñez, J. et al. (2007). Informe del Comité Científico de la Agencia Española de Seguridad Alimentaria y Nutrición (AESAN) sobre una cuestión planteada por la Dirección Ejecutiva de la AESAN, en relación con el riesgo de la posible presencia de $N$-nitrosaminas en productos cárnicos crudos adobados cuando se someten a tratamientos culinarios de asado o fritura. AESAN-2007-007. Recuperado de: http://aesan.msssi.gob.es/AESAN/docs/ docs/evaluacion_riesgos/comite_cientifico/ NITROSAMINAS_P.CARNICOS.pdf

Pérez, D.; Andújar, G. y Venegas, O. (2009). Química y bioquímica de la carne y los productos cárnicos. Instituto de Investigaciones para la Industria Alimentaria. Cuba: Editorial Universitaria. pp. 85-87 Recuperado de: http://site.ebrary.com. ezproxy.sibdi.ucr.ac.cr:2048/lib/sibdilibrosp/ reader.action? docID $=10317000$

Pérez, M.L., Bosch, N. y Garciá-Mata, M. (1996). Monitoring nitrite and nitrate residues in frankfurters during processing and storage. Meat Science. 44(1-2): 66-73.

Reglamento técnico rtcr: 411-2008 productos cárnicos embutidos: salchicha, salchichón, mortadela y chorizo. especificaciones. (2008). Recuperado de: http://reglatec.go.cr/reglatec/principal.js $\mathrm{p}$ ?irSeccion $=$ true $\&$ seccion $=268 \&$ padre $=166$

Sánchez, A., Torrescano, G.,Camou, J.P., Gónzalez, N. y Hernández, G. (2008). "Sistemas combinados de conservación para prolongar la vida útil de la carne y los productos cárnicos". Nacameh. 2(2): 124-159. 
Serrano-Pérez, D.; de Ona-Baquero, C. \& OrtsLaza, M. (2012). Elaboración de preparados cárnicos frescos: carnicería y elaboración de productos cárnicos (MF0297_2). España: IC Editorial. pp. 73, 81. Recuperado de: http://site. ebrary.com.ezproxy.sibdi.ucr.ac.cr:2048/lib/ sibdilibrosp/ reader.action?docID $=10721881$

Vindas, L. (2014). Embutidos generan fuerte competencia a otras carnes. El Financiero. Costa Rica. Recuperado de: http://www. elfinancierocr.com/negocios/embutidosCargill-Pipasa-Sigma_0_477552286.html 